\section{PREDICTING STI RISK AMONG PEOPLE ATTENDING SEXUAL HEALTH SERVICES: DEVELOPMENT OF A TRIAGE TOOL TARGETING BEHAVIOURAL INTERVENTIONS AMONG YOUNG PEOPLE}

${ }^{1}$ Carina King ${ }^{*},{ }^{1}$ Cath Mercer, ${ }^{2}$ Martina Furegato, ${ }^{2}$ Hamish Mohammed, ${ }^{1}$ Andrew Copas, ${ }^{1}$ Maryam Shahmanesh, ${ }^{1}$ Richard Gilson, ${ }^{2}$ Gwenda Hughes. ${ }^{1}$ Research Department of Infection and Population Health, University College London, London, UK; ${ }^{2}$ Department of HIV \& STIS, Centre for Infectious Disease Surveillance \& Control, Public Health England, London, UK

\subsection{6/sextrans-2016-052718.36}

Background/introduction There are very limited resources for delivering sexual health promotion within sexual health services (SHS).

Aim(s)/objectives Santé, a feasibility study for a trial of sexual risk reduction interventions, is developing a triage tool embedded within the electronic patient record to target interventions by risk score among young people (16-25 years) attending SHS.

Methods We used GUMCADv2, the national mandatory STI surveillance dataset (2013-2014 - Model 1), and the GUMCADv3 pilot (July-October 2015 - Model 2). Predictive logistic regressions for acute STI diagnosis were run. Model 1 only considered demographic and clinical variables; Model 2 also included enhanced behavioural data (number of partners, new partners, and condom use in the past 3 months).

Results 936,251 and 619 patient-episodes were included in Models 1 and 2 respectively, of which 11\% and 4\% involved an STI diagnosis. In Model 1, predicted risk of STI diagnosis ranged between $1-47 \%$ (pseudo- $\mathrm{R}^{2}$ : 1.9\%). Referring the riskiest (highest decile) patients to more intensive interventions gives a sensitivity and specificity of $70 \%$ and $45 \%$, respectively, and a positive predictive value (PPV) of $13 \%$ for STI diagnosis. In Model 2 the predicted risk of STI was $0-53 \%$ (pseudo- $\mathrm{R}^{2}$ : $23 \%)$, and referring the riskiest patients demonstrated an improved sensitivity (76\%), specificity (87\%) and PPV (25\%).

Discussion/conclusion Routinely collected surveillance data can be used to triage young people for targeted risk-reduction interventions, but this is more robust if behavioural data are taken into account. Addition of behavioural data to routine STI surveillance (GUMCADv3) is a powerful way to target sexual health promotion.

\section{THE NATIONAL HIV SELF-SAMPLING SERVICE}

${ }^{1}$ Luis Guerra*, ${ }^{1}$ Louise Logan, ${ }^{2}$ Tim Alston, ${ }^{2}$ Noel Gill, ${ }^{2}$ Ryan Kinsella, ${ }^{1}$ Anthony Nardone. ${ }^{1}$ Public Health England, London, UK; ${ }^{2}$ Preventx Integrated Diagnostics, Sheffield, UK

10.1136/sextrans-2016-052718.37

Background/introduction In November 2015, Public Health England, with the support of Local Authorities, launched a nationwide HIV self-sampling service free for populations most at-risk of HIV acquisition (www.freetesting.hiv). In February 2016 the service was devolved to participating local authorities who have taken responsibility for the service in their areas.

$\operatorname{Aim}(\mathbf{s}) /$ objectives To determine who is accessing the service and whether it reached most at-risk groups (including MSM and Black African communities) and first-time testers.

Methods Disaggregated anonymised data from service users ordering kits from 18 November 2015 - 31 January 2016 were analysed, including: ethnicity, gender, sexual orientation, local authority residency and self-reported HIV testing information.
Results During this period there were 17,114 kits ordered of which $51 \%(\mathrm{n}=8,706)$ were returned with a $1.4 \%$ reactive rate $(\mathrm{n}=122) .82 \%(\mathrm{n}=7149)$ of kits returned were from MSM with a $1.34 \%$ reactive rate $(n=96) .32 \%$ reported never testing and $40 \%$ testing over a year ago. $18 \%(\mathrm{n}=1537)$ of kits returned were from heterosexuals. Of those $42 \%(n=649)$ were from Black African individuals with a $1.54 \%$ reactive rate $(\mathrm{n}=10)$ and $31 \%$ reported never testing and $45 \%$ testing over a year ago. Manchester, Leeds and Birmingham are the local authorities presenting the highest service demand across England.

Discussion/conclusion The national self-sampling service has been successful at engaging most at-risk populations for HIV acquisition across the nation and those who had not tested for HIV as frequently as recommended in national guidelines; including many who have never tested before.

\section{ROLE OF PRIMARY CARE IN THE DIAGNOSIS OF STIS IN ENGLAND}

Emma Beaumont*, Martina Furegato, Hamish Mohammed, Gwenda Hughes. Public Health England, London, UK

\subsection{6/sextrans-2016-052718.38}

Background Sexually transmitted infection (STI) diagnoses made in genitourinary medicine (GUM) clinics have been collected in England for many years, but little is known about the contribution of GPs to STI diagnoses.

Objectives To assess trends in diagnosis rates of selected STIs from GPs.

Methods Longitudinal analysis of age- and sex-standardised population diagnosis rates of selected STIs from GPs in England from 2005-2014 was performed using data from the Clinical Practice Research Datalink (CPRD).

Results The proportion STI diagnoses made by GPs varied by infection, ranging from 2\% (gonorrhoea) to 34\% (genital herpes), in 2014. From 2005-2014, diagnosis rates [95\% CI] per 100,000 registered population decreased for chlamydia (51.4 [50.7-52.0] to 24.9 [24.5-25.3], $\mathrm{p}=0.009)$, gonorrhoea $(3.4$ [3.2-3.5] to $1.8[1.7-1.9], \mathrm{p}=0.02)$, genital warts $(73.1$ [72.473.9] to $38.4,[37.9-39.0], \mathrm{p}=0.004)$ and genital herpes $(36.9$ [36.4-37.4] to 26.1 [25.7-26.6], p = 0.02). Diagnosis rates for all four STIs were higher among women, particularly for chlamydia and genital herpes where respective 2014 rates were 38.3 [37.6-39.1] and 41.8 [41.0-42.5] compared to 11.1 [10.7-11.5] and 10.0 [9.7-10.4] in men.

Conclusion While the rates of STI diagnoses in GUM clinics in England have steadily risen in the past 10 years, particularly in men, diagnosis rates of these four STIs in GPs have decreased between 2005 and 2014. The high diagnosis rates seen in women in GPs, suggest that primary care has an important role in the diagnosis of STIs in women although their relative contribution may have declined in recent years.

\section{O040 A PARTNER NOTIFICATION BUREAU IN ACTION: OUTCOMES FOR CENTRALISED MANAGEMENT OF POSITIVE GONORRHOEA AND CHLAMYDIA RESULTS FROM PRIMARY CARE BY A SEXUAL HEALTH SERVICE}

Gill Bell. Sheffield Teaching Hospitals NHS Foundation Trust, Sheffield, Yorkshire, UK

10.1136/sextrans-2016-052718.39 